\title{
Treatment of periodontal intrabony defects using autologous periodontal ligament stem cells: a randomized clinical trial
}

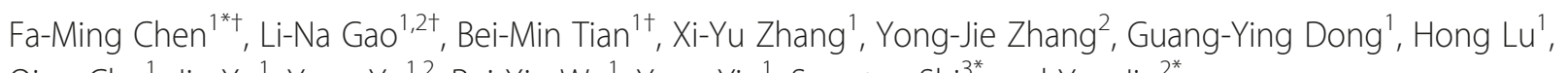
Qing Chu', Jie Xu', Yang Yu ${ }^{1,2}$, Rui-Xin $\mathrm{Wu}^{1}$, Yuan Yin ${ }^{1}$, Songtao Shi ${ }^{3^{*}}$ and Yan $\mathrm{Jin}^{2^{*}}$

\begin{abstract}
Background: Periodontitis, which progressively destroys tooth-supporting structures, is one of the most widespread infectious diseases and the leading cause of tooth loss in adults. Evidence from preclinical trials and small-scale pilot clinical studies indicates that stem cells derived from periodontal ligament tissues are a promising therapy for the regeneration of lost/damaged periodontal tissue. This study assessed the safety and feasibility of using autologous periodontal ligament stem cells (PDLSCS) as an adjuvant to grafting materials in guided tissue regeneration (GTR) to treat periodontal intrabony defects. Our data provide primary clinical evidence for the efficacy of cell transplantation in regenerative dentistry.
\end{abstract}

Methods: We conducted a single-center, randomized trial that used autologous PDLSCs in combination with bovine-derived bone mineral materials to treat periodontal intrabony defects. Enrolled patients were randomly assigned to either the Cell group (treatment with GTR and PDLSC sheets in combination with Bio-oss') or the Control group (treatment with GTR and Bio-oss ${ }^{\circ}$ without stem cells). During a 12-month follow-up study, we evaluated the frequency and extent of adverse events. For the assessment of treatment efficacy, the primary outcome was based on the magnitude of alveolar bone regeneration following the surgical procedure.

Results: A total of 30 periodontitis patients aged 18 to 65 years (48 testing teeth with periodontal intrabony defects) who satisfied our inclusion and exclusion criteria were enrolled in the study and randomly assigned to the Cell group or the Control group. A total of 21 teeth were treated in the Control group and 20 teeth were treated in the Cell group. All patients received surgery and a clinical evaluation. No clinical safety problems that could be attributed to the investigational PDLSCs were identified. Each group showed a significant increase in the alveolar bone height (decrease in the bone-defect depth) over time $(p<0.001)$. However, no statistically significant differences were detected between the Cell group and the Control group $(p>0.05)$.

Conclusions: This study demonstrates that using autologous PDLSCs to treat periodontal intrabony defects is safe and does not produce significant adverse effects. The efficacy of cell-based periodontal therapy requires further validation by multicenter, randomized controlled studies with an increased sample size.

(Continued on next page)

\footnotetext{
* Correspondence: cfmsunhh@fmmu.edu.cn; songtaos@dental.upenn.edu; yanjin@fmmu.edu.cn

${ }^{\dagger}$ Equal contributors

'State Key Laboratory of Military Stomatology, Department of

Periodontology, School of Stomatology, Fourth Military Medical University,

Xi'an, Shannxi, P. R. China

${ }^{3}$ Department of Anatomy and Cell Biology, School of Dental Medicine,

University of Pennsylvania, 240 South 40th Street, Philadelphia, PA 19104, USA

${ }^{2}$ State Key Laboratory of Military Stomatology, Research and Development

Center for Tissue Engineering, School of Stomatology, Fourth Military Medical

University, Xi'an, Shannxi, P. R. China
} 
(Continued from previous page)

Trial Registration: NCT01357785 Date registered: 18 May 2011.

Keywords: Stem cell-therapy, Periodontitis, Periodontal regeneration, Cell sheet, Tissue engineering, Translational medicine

\section{Background}

Periodontitis is an inflammatory disease that causes pathological alterations in tooth-supporting tissues, which can lead to tooth loss if left untreated. National surveys have shown that the majority of adults suffer from moderate periodontitis, and up to $15 \%$ of the population is affected by severe generalized periodontitis at some stage of their lives $[1,2]$. The significant burden of periodontal disease and its impact on general health and patient quality of life suggest a clinical need for the effective management of this condition [3-5]. The ultimate goal of periodontal therapy is the predictable regeneration of the functional attachment apparatus that is destroyed by periodontitis, which involves at least three unique tissues, including the cementum, periodontal ligament (PDL), and alveolar bone. To date, several regenerative procedures have been developed in an attempt to treat periodontitis, including guided tissue regeneration (GTR), bone graft placement, and the use of bioactive agents, such as growth factors (reviewed in [5-8]). However, the current therapeutic techniques used either alone or in combination have limitations in producing complete and predicable regeneration, especially in advanced periodontal defects. In these cases, remaining deep intraosseous defects following periodontal therapy are high-risk sites for the further progression of periodontitis (reviewed in [9-11]).

According to histological evidence, the GTR technique combined with grafting materials, such as Bio-oss (Geistlich Pharm. AG, Volhusen, Switzerland) and autologous bone, is partially effective at treating periodontal defects; however, the currently available GTR-based therapies remain rudimentary and show poor clinical predictability (reviewed in $[7,8,11]$ ). Recent advances in stem cell biology and regenerative medicine have enabled the use of cell-based therapy in periodontal diseases (reviewed in $[5,12])$. To date, a large number of studies have indicated that ex vivo-manipulated stem cells derived from either bone marrow or the PDL can be used in conjunction with different physical matrices (autografts, xenografts, allografts, and alloplastic materials) to regenerate periodontal tissues in vivo (reviewed in [13-15]).

Although controversy remains regarding which tissues provide the most appropriate donor source for cell isolation, there is evidence that the cells of PDL tissues have the capacity to form a complete periodontal attachment apparatus (reviewed in [13-15]). The regenerative capacity of the PDL is attributed to a few progenitor cells within the PDL that maintain their proliferation and differentiation potential; thus, regeneration of the periodontium depends on the participation of these mesenchymal stem/stromal cells (MSCs) (reviewed in [5, 12, 13]). PDL-derived progenitors are committed to several developmental lineages, i.e., osteoblastic, fibroblastic and cementoblastic [16], which suggests that these cells are capable of regenerating multiple periodontal tissues. Indeed, positive preclinical results have been achieved in a wide range of in vitro and in vivo models [17-28]. The next phase of study requires the clinical application of these advanced therapies.

Worldwide, periodontitis remains highly prevalent and leads to a loss of the affected teeth. This disease threatens the quality of life of the middle-aged population as far as oral functioning is concerned. Unfortunately, no current clinical periodontal treatments can heal the defects in the affected region or regenerate lost periodontal tissue to a normal structure and functionality. It is clear that there is a clinical need for such treatments and a vast patient demand. Importantly, several groups have commenced small-scale pilot/feasibility studies in humans [21, 29-31]; thus, there is now sufficient information to support endeavors to move cell-based periodontal therapy into the clinical arena. We established a clinical protocol to further test the safety, feasibility, and potential efficacy of stem cells for the treatment of periodontal deep intraosseous defects.

\section{Methods \\ Study design}

This study had a randomized design involving one dental facility (Translational Research Center, School of Stomatology, Fourth Military Medical University) and was conducted in compliance with Good Clinical Practice (GCP) guidelines according to the schedule shown in Fig. 1. This clinical trial, including the recruitment of subjects, was performed from 1 June 2011 to 30 December 2013, and the study was completed at the end of 2014 with a 1-year follow-up of the patients. The study protocol for this trial is provided in Additional file 1.

\section{Ethics}

This study was approved by the ethical committees of the School of Stomatology, Fourth Military Medical University (2011-02) and is registered with the ClinicalTrials.gov database (reference no. NCT01357785). This study was conducted according to the Declaration of Helsinki, and 


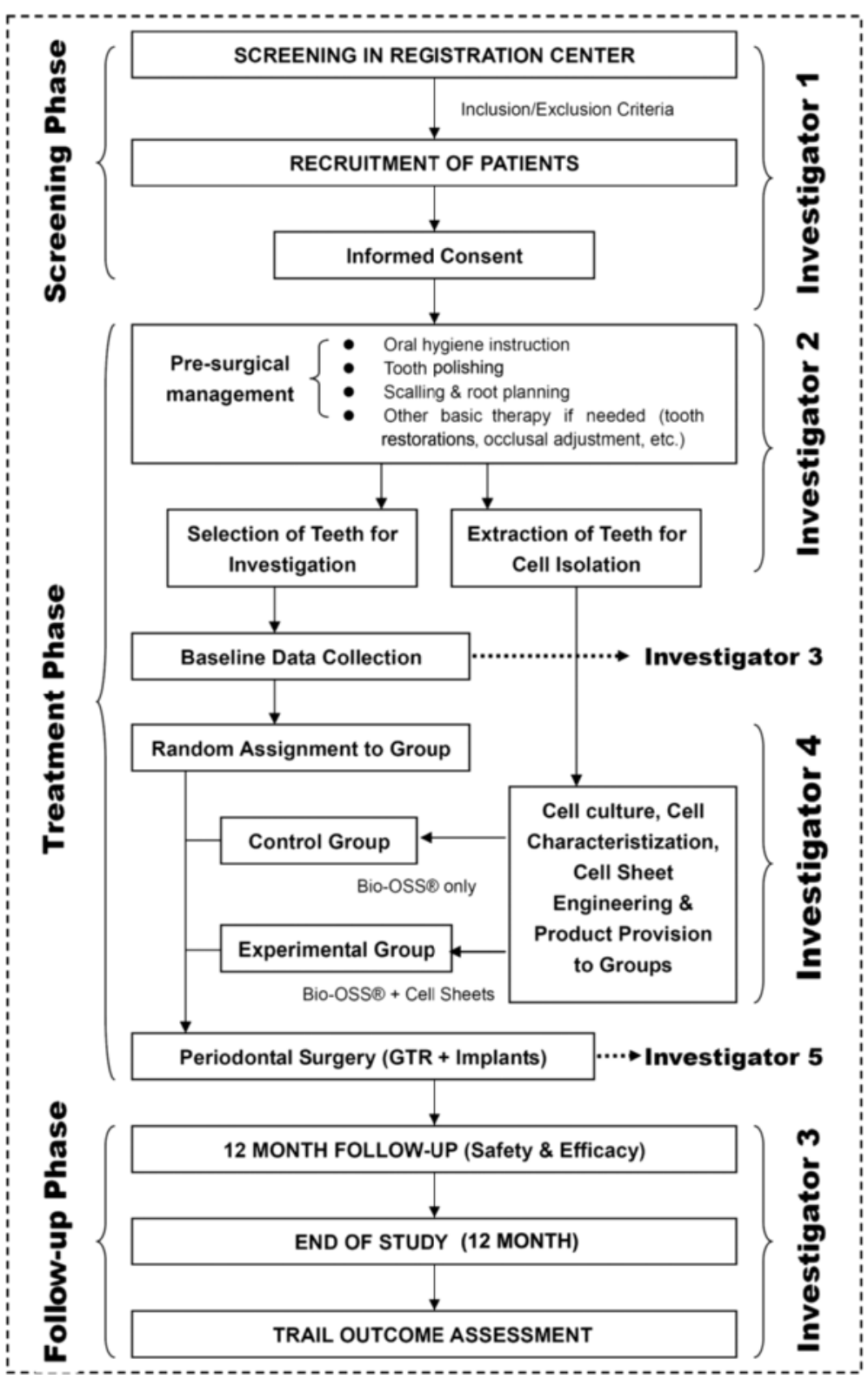

Fig. 1 The flow chart of the trial describes the selection, randomization, treatment and follow-up process. A physician (Investigator 3) performed the follow-up examination of the patients and remained blinded to the treatment conditions until the clinical trial was completed. GTR Guided tissue regeneration

all recruited patients consented to participate in this trial and contribute their trial data for noncommercial purposes. The protocol of this trial was externally reviewed and approved by an anonymous independent ethical review committee to ensure no serious ethical concerns.

\section{Patients, enrollment and randomization}

Patients with periodontitis visiting our dental institution were requested to participate in the study. In compliance with GCP guidelines, prospective patients who provided written informed consent underwent clinical inspection and an oral cavity diagnosis. We selected subjects who satisfied the inclusion and exclusion criteria (recorded as the date of recruitment). The majority of these criteria were used in previous similar periodontal clinical trials $[32,33]$. The inclusion and exclusion criteria and methods for randomization are provided in Additional file 2 (Appendices 1 and 2).

\section{Study products and interventions}

The third molars of the patients in the Cell group were extracted and subjected to cell isolation and transplant 
production according to the Good Laboratory Practice and Good Manufacturing Practice (GMP) guidelines. The cells were assessed for cell colony-forming ability and osteogenic/adipogenic differentiation (Fig. 2A). Prior to the extraction surgery, at least two independent assessors concluded that a tooth (or teeth) extraction was required due to impacted or nonfunctional reasons. The methods for cell isolation and characterization are presented in Additional file 2 (Appendix 3). The PDL cell sheets obtained from the patient's own tooth/teeth (see inclusion criteria) were produced using the Good Laboratory Practice and GMP guidelines using a standardized procedure in the Research and Development Center for Tissue Engineering (Fourth Military Medical University, 145th West Chang-le Road, Xi'an 710032, Shaanxi, People's Republic of China). The detailed method is described in Additional file 2 (Appendix 3). Bio-Oss and Bio-Guide were purchased from Geistlich Pharma AG (Volhusen, Switzerland). Both transplants (Bio-oss only or Bio-oss /cell sheets) were freshly prepared by laboratory researchers (Fig. 2B a-c). Investigator 3 , who performed the follow-up study, was kept blinded to the treatment conditions until the study was completed. For the surgical treatment, Bio-oss only (Control group) or Bio-oss /cell sheets (Cell group) were administered only to the bony defect region (Fig. 2B d-f). Each subject received a standard initial preparation, including oral hygiene instruction, full-mouth scaling, and root planning before surgical treatment, in order to minimize the bacterial insult and reduce variability between lesions at baseline. The operations were performed using GCP procedures. A 12-month postoperative follow-up was performed for each patient.

\section{Safety assessment}

A cell safety assessment was performed to detect chromosomal karyotype changes between freshly isolated periodontal ligament stem cells (PDLSCs) and those obtained from the cell sheets (the latter underwent approximately 30-day ex-vivo cultures). The detailed methods are described in Additional file 2 (Appendix 3). Complications and adverse events during postoperative healing were recorded, and we examined the extent of adverse event occurrence. In addition, blood was obtained from patients preoperatively and at 2 weeks, 3 months and 12 months postoperatively. Blood examinations included: (i) a decrease in the white blood cell count; (ii) an increase in the red blood cell count; (iii) a decrease/ increase in the percentage of neutrophils; (iv) a decrease/increase in the percentage of lymphocytes; (v) an increase in blood bilirubin; (vi) a decrease in blood lactate dehydrogenase; (vii) an increase in C-reactive protein; and (viii) an increase in creatinine phosphokinase. Moreover, the levels of IgA, IgG, IgM, C3 and C4

A

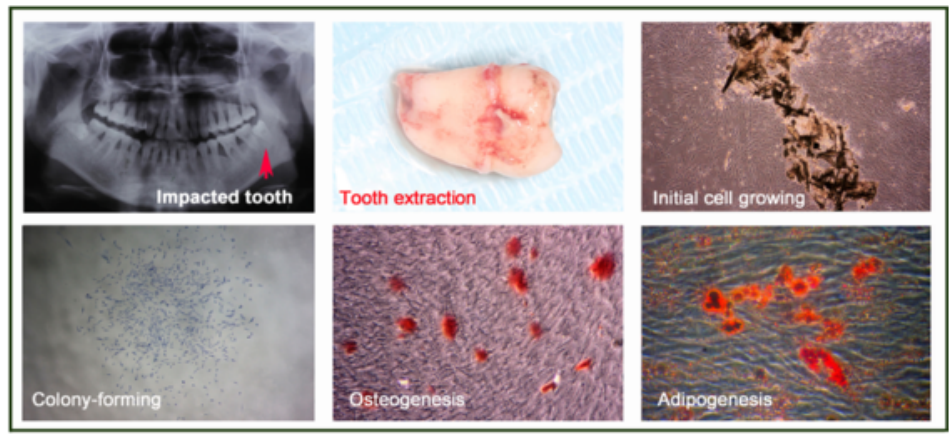

B

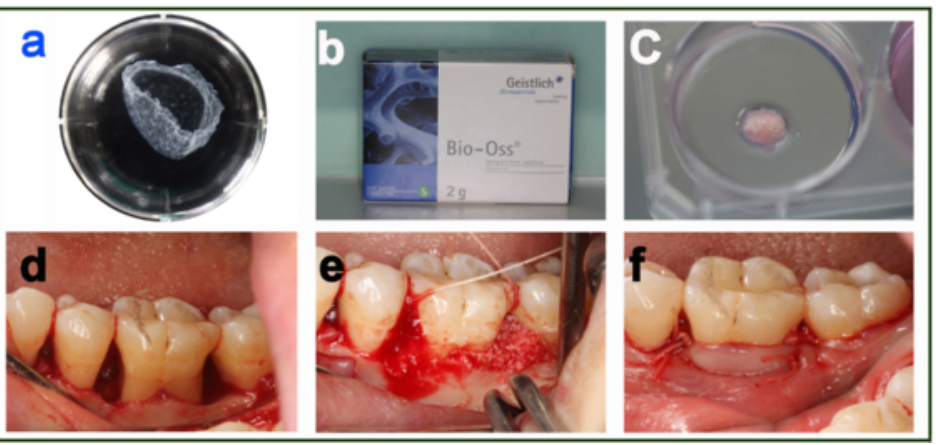

Fig. 2 Cell isolation, characterization and surgery. A The impacted third molar of patients was extracted and subjected to cell isolation and cell characterization for cell colony-forming ability and osteogenic/adipogenic differentiation. B The production of cell sheet/scaffold transplants and in vivo transplantation, including: (a) cell sheet formation; (b) Bio-Oss particulates; (c) cell sheet/scaffold transplants; (d) exposure of bone defects; (e) placement of transplants; and ( $f$ ) closure of the flap 
were measured in the serum using enzyme-linked immunosorbent assay (ELISA) at the Department of Clinical Laboratory, Fourth Military Medical University School of Stomatology. At the time of blood collection, the urine of each patient was collected and assessed for: (i) a positive test for glucose/albumin; (ii) an increase in $\beta$-N-acetyl- $D$-glucosaminidase; and (iii) an increase in $\beta 2$ microglobulin.

\section{Efficacy assessment}

The main outcome measure in the study protocol was the rate of increase in alveolar bone height at 3, 6 and 12 months postoperation (primary outcome). The bone-defect depth (the distance in millimeters from the deepest part of the defect to the cementoenamel junction of the tooth) was measured as described in Additional file 2 (Appendix 4) [34]. The clinical attachment level (CAL), probing depth (PD) and gingival recession (GR) measured in millimeters are generally used to assess pathology in periodontal disease. However, these parameters do not directly assess the efficacy of cells in periodontal tissue regeneration and were selected as secondary outcome measures to ascertain if the cells caused abnormal periodontal healing following periodontal surgery. The methods for the determination of these parameters at baseline and 3 months postoperation are described in Additional file 2 (Appendix 4) [32, 33].

\section{Statistics}

This study was performed using a per-protocol analysis. In this analysis, all of the randomized teeth received at least one therapy, but the teeth that did not receive treatment were excluded (modified per-protocol analysis). The last-observation-carried-forward method was used for the per-protocol analysis. The missing data points were input into the postbaseline follow-up visits from the last observation available for each patient. For analysis, we employed SAS version 8.2 software (SAS Institute Inc., Carey, North Carolina, USA). The perprotocol set analysis was performed for the primary outcome. The baseline between-group comparisons in age and clinical examination indices were performed using independent group $t$ tests. The between-group comparison of sex was performed using the Fisher's exact probability test. The changes in clinical examination indices were tested using a repeated-measures analysis of variance. The level of statistical significance was set at $p<0.05$ prior to analysis.

\section{Results}

\section{Enrollment and teeth}

The flow diagram for the study is shown in Fig. 3. A total of 48 screened teeth were randomly assigned to either the Control group or the Cell group. However, only 41 teeth received surgery (21 teeth in the Control group and 20 teeth in the Cell group). The baseline measurements of the teeth are shown in Table 1. A Fisher's exact probability test found no significant between-group differences in the donors who provided teeth for randomization and testing.

\section{Cell culture and surgery}

In this trial, patients who had at least one tooth (e.g., wisdom tooth) that needed to be extracted due to impaction or nonfunctional reasons and agreed to the tooth extraction were enrolled. Prior to extraction surgery, at least two independent assessors concluded that a tooth or teeth required extraction. The extracted teeth were used for cell isolation. Only two teeth failed during the cell isolation step, and the corresponding two patients were excluded from further study. All of the cells exhibited colony-forming ability. In addition, these cells were positive for the MSC markers STRO-1, CD146, CD105, CD29, and CD90 and negative for the hematopoietic markers CD31 and CD45. The cells were successfully differentiated in osteogenic and adipogenic microenvironments and subsequently used for cell sheet production and periodontal surgery (refer to Additional file 2 (Appendix 3) for more information).

\section{Safety evaluation}

Postoperative healing occurred without significant problems, and none of the patients reported any complications/adverse events other than medium-sized swelling and pain. None of the pain experienced by patients required therapy. All of the patients underwent blood and urine tests preoperatively and at 2 weeks, 3 months and 12 months postoperatively. Changes in the white/red blood cell count, percentage of neutrophils/lymphocytes, and blood bilirubin/lactate dehydrogenase/C-reactive protein/creatinine phosphokinase levels were within the clinically accepted range (no measurement exceeded its clinical reference value). Importantly, no significant changes in IgA, IgG, IgM, C3 or C4 concentrations were found in the serum of any of the patients. A urine test showed that one patient (with one tooth that received GTR and Bio-oss therapy without stem cells) was positive for glucose (this patient was ultimately not diagnosed with diabetes mellitus) and two patients were positive for albumin (each patient had two teeth involved in this trial, and one tooth per patient received cell therapy). No significant changes in urinary $\beta-\mathrm{N}$ acetyl- $D$-glucosaminidase or $\beta 2$ microglobulin were found for any of the patients.

\section{Evaluation of efficacy}

Patient demographic data and the baseline measurements of the affected teeth are shown in Table 1. All of the treated teeth in both groups adequately recovered 
$N$ : No. of patients

$n$ : No. of teeth

CAL: clinical attachment levels

PD: probe depth (pocket depths)

GR: gingival recession levels

* alveolar bone height
Assessed for eligibility $(N=50)$

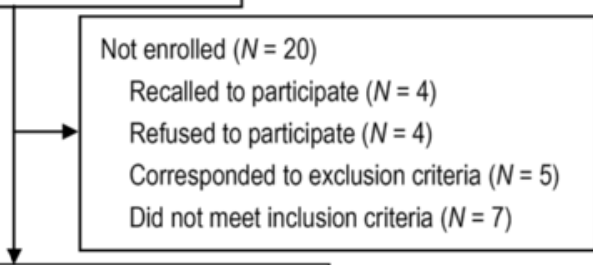

Participants enrolled $(N=30)$

Eligible teeth $(n=48)$

Randomized

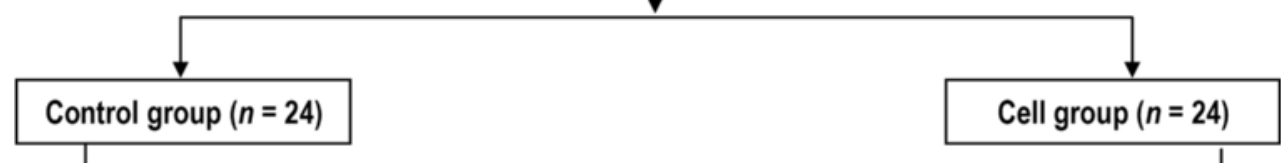

Aborted $(n=3)$

Not eligible bone defect $(n=2)$

Recalled before surgery $(n=1)$

Received surgery

( $n=21$ )

Aborted ( $n=4)$

Not eligible bone defect $(n=2)$

Failed in cell isolation $(n=2)$

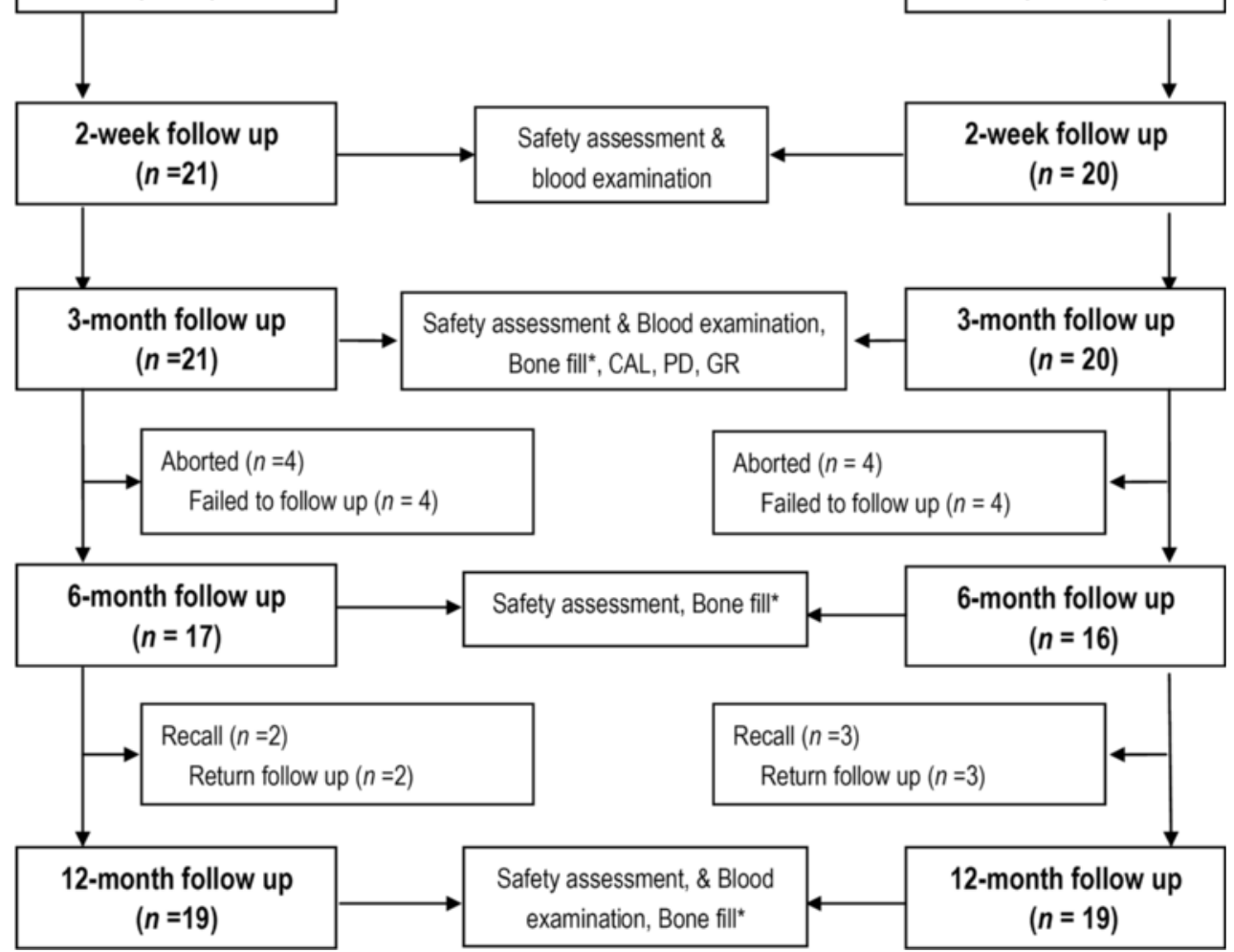

Fig. 3 The flow diagram of the trial 
Table 1 Teeth and baseline

\begin{tabular}{|c|c|c|c|}
\hline & Control group & Cell group & $p$ value \\
\hline$\overline{\operatorname{Sex}(n)}$ & 21 & 20 & $0.134^{\mathrm{a}}$ \\
\hline Male $(n)$ & 6 & 2 & \\
\hline Female $(n)$ & 15 & 18 & \\
\hline Age (years, mean $\pm \mathrm{SE}$ ) & $30.04 \pm 7.90$ & $26.05 \pm 4.44$ & $0.053^{\mathrm{b}}$ \\
\hline $\mathrm{CAL}(\mathrm{mm}$, mean $\pm \mathrm{SE})$ & $5.28 \pm 1.60$ & $5.15 \pm 1.52$ & $0.795^{\mathrm{b}}$ \\
\hline $\mathrm{BDD}(\mathrm{mm}$, mean $\pm \mathrm{SE})$ & $7.19 \pm 1.87$ & $7.20 \pm 2.65$ & $0.990^{\mathrm{b}}$ \\
\hline \multicolumn{4}{|l|}{$\mathrm{PD}(\mathrm{mm}$, mean $\pm \mathrm{SE})$} \\
\hline Facial & $5.68 \pm 1.59$ & $6.43 \pm 1.92$ & $0.185^{\mathrm{b}}$ \\
\hline Lingua (palatal) & 5. $86 \pm 1.43$ & $6.25 \pm 1.36$ & $0.373^{b}$ \\
\hline \multicolumn{4}{|c|}{ GR (mm, median (interquartile range)) } \\
\hline Facial & $0.33(1.0)$ & & $0.692^{c}$ \\
\hline Lingua (palatal) & $0.33(0.83)$ & & $0.320^{c}$ \\
\hline
\end{tabular}

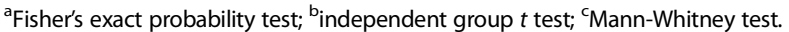
$B D D$ bone-defect depth, CAL clinical attachment levels, GR gingival recession, $P D$ probe depth, SE standard error

following surgery. There was no loss of treated teeth during this trial. X-ray examinations showed significant bone fill in both groups (Fig. 4). The magnitude of increase in alveolar bone height at 3, 6 and 12 months (bone fill over time) was determined as the decrease in the bone-defect depth. Each group showed a significant increase in the alveolar bone height over time $(p<0.001)$. However, no statistically significant differences were found between the Cell group and the Control group ( $p>0.05)$ (Table 2). Regarding the clinical periodontal parameters, no statistically significant differences were found for the increased CAL, PD or GR between the Cell and Control groups at 3 months postsurgery $(p>0.05)$ (Table 3$)$.

\section{Discussion}

Although there are a number of clinical techniques available for the management of periodontal intrabony defects, clinicians continue to seek more predictable regenerative therapies that are less technique-sensitive, lead to rapid tissue regeneration, and applicable to the broad array of periodontal conditions that are encountered daily in the clinic. Recent evidence from animal models [17-28] and several small-scale pilot/feasibility studies [21, 29-31] indicates that ex vivo-cultured PDL cells may serve as a powerful tool for periodontal therapy. A number of animal studies have provided an overwhelming body of evidence that MSCs can be safely and effectively used for periodontal regeneration (reviewed in [12]). As a consequence of these successful animal studies, the clinical application of

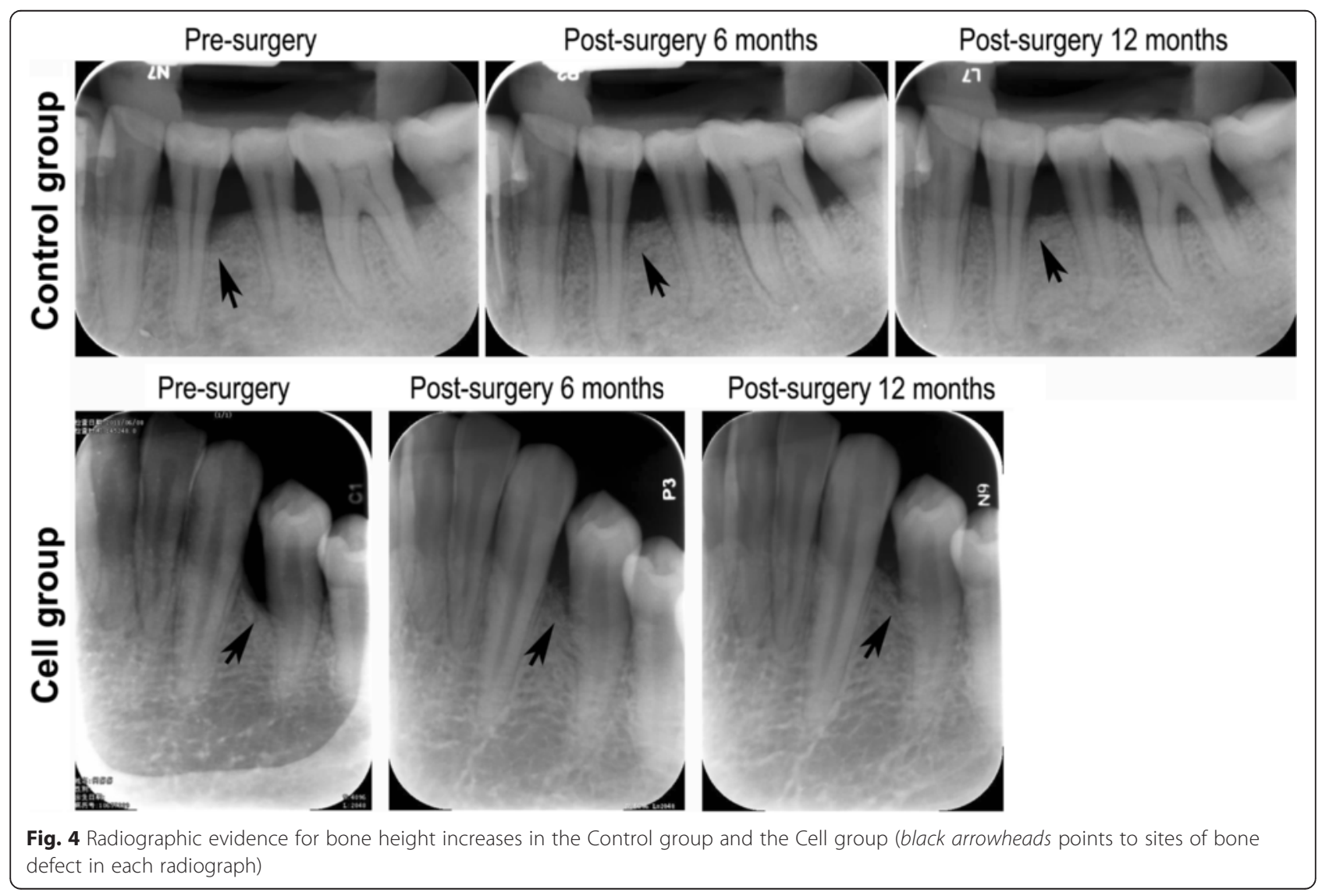


Table 2 Bone-defect depth with time (the distance from the deepest part of the defect to the cementoenamel junction of the tooth in $\mathrm{mm}$, mean \pm standard error)

\begin{tabular}{|c|c|c|c|c|c|c|c|}
\hline & No. of teeth & Baseline & 3 months & 6 months & 12 months & $F$ value & $p$ value \\
\hline Control group & 21 & $7.19 \pm 1.87$ & $4.81 \pm 1.93$ & $5.11 \pm 1.53$ & $4.80 \pm 1.41$ & 0.11 & $0.742^{a}$ \\
\hline Cell group & 20 & $7.20 \pm 2.65$ & $4.89 \pm 1.73$ & $4.61 \pm 1.87$ & $4.49 \pm 2.03$ & & \\
\hline
\end{tabular}

${ }^{a}$ Repeated-measures analysis of variance

stem cells for the regeneration of periodontal tissue has begun [30, 31].

Substantial evidence suggests that it is time is to move cell-based periodontal therapy from animal studies to human clinical trials. However, there are critical steps in moving this field towards human clinical utility. In addition to clinical efficacy, the safety of cell-based therapies has not been fully evaluated, and the risks of stem cell therapies have been underscored by several clinicians and researchers. Moreover, issues, such as cell delivery, cell immunogenicity, use of autologous cells or allogeneic cells, control of cell fates in vitro and in vivo, and cost-effectiveness, are all important considerations that should be addressed before this therapy can move forward [35-39]. The next critical phase requires the identification of tissues that provide the most appropriate donor source(s) and the systematic validation of these specific MSCs as reliable for periodontal cytotherapeutic use. Furthermore, the establishment of large-scale preparation facilities incorporating the stringent protocols of GMP will be an absolute necessity. Regulatory agencies need to define new criteria to evaluate the risk associated with specific stem cells and their differentiated progeny (reviewed in $[35,36]$ ). The purpose of this trial was to provide evidence for the use of ex vivo-cultured cells to treat periodontitis and determine the best approach to treat this disease.
For incurable and life-threatening diseases, such as diabetes, Parkinson's, muscular dystrophy, Alzheimer's, neural and cardiac diseases and refractory systemic lupus erythematosus, cell-based therapy is more likely to be warranted and accepted by the government and patients [37-39]. However, periodontal tissue regeneration using cell therapy is not yet economically viable or competitive with current root canal therapies and dental implants. Due to the non-life threatening nature of periodontitis, periodontal tissues have not been considered to be a major target for stem cell-based regenerative medical research. Nevertheless, affected teeth are ideal for the evaluation of new therapies because the patients are not usually ill. Thus, if anything goes wrong with the treatment, the situation is far less likely to be life threatening. Furthermore, the accessibility of teeth facilitates treatments that do not require major surgery [40].

In a previous study by Feng et al., periodontitis patients were treated with the local administration of autologous PDL or gingival stem cells. Neither adverse reactions nor an increase in any autoantibodies was observed; however, only three cases were observed long-term [30]. To move cell-based periodontal therapy from preclinical study and case report into clinical trials, we conducted this study to evaluate the safety and primary efficacy of this treatment. The results of this study have implications for oral health care resourcing and may facilitate improvements in the

Table 3 Changes in clinical examination indices over time ( $\mathrm{mm}$, mean \pm standard error)

\begin{tabular}{|c|c|c|c|c|c|c|}
\hline & No. of teeth & & Baseline & 3 months & $F$ value & $p$ value \\
\hline \multicolumn{7}{|l|}{ CAL } \\
\hline Control group & 21 & & $5.28 \pm 1.60$ & $5.07 \pm 1.48$ & 0.817 & $0.371^{a}$ \\
\hline Cell group & 20 & & $5.15 \pm 1.52$ & $4.42 \pm 1.19$ & & \\
\hline \multicolumn{7}{|l|}{ PD } \\
\hline Control group & 21 & Buccal & $5.68 \pm 1.59$ & $3.88 \pm 0.77$ & 0.962 & $0.333^{\mathrm{a}}$ \\
\hline Cell group & 20 & Buccal & $6.43 \pm 1.92$ & $3.80 \pm 1.03$ & & \\
\hline Control group & 21 & Lingual or palatal & $5.86 \pm 1.43$ & $3.79 \pm 0.55$ & 2.191 & $0.147^{\mathrm{a}}$ \\
\hline Cell group & 20 & Lingual or palatal & $6.25 \pm 1.36$ & $4.20 \pm 0.86$ & & \\
\hline \multicolumn{7}{|l|}{ GR } \\
\hline Control group & 21 & Buccal & $0.62 \pm 0.89$ & $1.54 \pm 0.96$ & 0.133 & $0.728^{a}$ \\
\hline Cell group & 20 & Buccal & $0.70 \pm 1.09$ & $1.28 \pm 0.82$ & & \\
\hline Control group & 21 & Lingual or palatal & $0.52 \pm 0.85$ & $1.38 \pm 1.37$ & 0.012 & $0.915^{a}$ \\
\hline Cell group & 20 & Lingual or palatal & $0.73 \pm 0.87$ & $1.23 \pm 0.92$ & & \\
\hline
\end{tabular}

${ }^{a}$ Repeated-measures analysis of variance. CAL clinical attachment levels, $P D$ probe depth, GR gingival recession 
treatment of people with periodontitis. No clinical safety problems attributable to the investigational PDLSCs were identified in our study. Each group showed a significant increase in alveolar bone height over time $(p<0.001$; Table 2). Although no statistically significant differences were found for the outcomes in the bone and clinical parameters between the Cell and Control groups $(p>0.05$; Tables 2 and 3), the results of this study were the first to show that cell-based interventions are safe for human dental use in trials. Geistlich Bio-Oss is a natural bone substitute with osteoconductive properties; however, there is no substantial evidence that this product will lead to effective or predictable bone regeneration in periodontal intrabony defects. Because Bio-Oss particles may become an integral part of the newly formed bone framework and preserve volume over time [41], the use of this material as a scaffold for PDLSCs requires further investigation. Previous studies showed that allogeneic bone matrix may regenerate new bone, new cementum, and a new PDL around teeth that were previously contaminated by bacterial plaque $[42,43]$. Human-derived biomaterials would be the first choice as a cell carrier in similar future trials [44]. Nevertheless, the results that were obtained in this study may have important implications for the design of more appropriate cell-delivery materials to test cell therapies in patients with periodontitis.

The study presented here used central randomization, which is a strict and complete randomization method that ensures adequate concealment. The surgeon and the investigator who collected the baseline and follow-up data worked independently in this trial. Throughout the entire trial, the patients were not aware of which group they were assigned to. The patients were only informed that they would receive a periodontal surgical treatment that potentially included cell products. This trial was a singlecenter, randomized controlled study of 30 patients with 12 months of follow-up. In a phase I clinical trial, safety assessments should be the primary outcome. The methodology in this study was designed to assess both safety and efficacy because the use of PDL-derived cells for periodontal therapy was previously reported several years ago [30]. Indeed, several groups worldwide have completed smallscale pilot/feasibility studies that indicated no adverse reactions after local cell administration [29, 31]. Although these studies did not have a randomized designed, the reported safety of the treatment offers substantial evidence to justify the use of cell-based periodontal therapy in the clinic. To date, this study was one of the largest randomized controlled trials addressing the safety and effectiveness of cell therapy in combination with GTR and bone replacement for periodontitis. However, this study does not deliver substantial evidence to confirm the safety of cell-based periodontal therapy. Similar to the clinical use of stem cells in the oral cavity (excluding the periodontium) for bone regeneration [45, 46], cell-based techniques in periodontal regenerative medicine should be further investigated in more challenging clinical scenarios with well-designed and standardized randomized controlled trials. In addition, these therapies should be tested in combination with bioactive molecules and new materials in an attempt to improve the final outcome. We plan to design a phase II clinical trial for cell-based periodontal therapy aimed at selecting more suitable scaffolding materials, determining appropriate cell doses, and providing evidence for multicenter, randomized controlled trials.

\section{Conclusions}

Stem cell therapy is a promising new therapeutic avenue that may enable the regeneration of lost periodontal tissue, and regenerative dentistry is at the forefront of the transition from basic science research to the clinical reconstructive arena. Although there are many issues that need to be resolved before stem cell therapies become commonplace, clinicians should continue to monitor the progression of these technologies. The data obtained in this study showed that autologous PDLSC-based treatment for periodontal intrabony defects was safe; however, more rigorous clinical trials are recommended to evaluate the efficacy of this therapy. Future clinical endeavors in cellbased periodontal therapy should identify more suitable scaffolding materials and define safe and effective cell dosing procedures based on well-designed, multicenter, randomized controlled trials.

\section{Consent to publish}

The authors confirm that they have obtained consent from the participants to publish the trial data.

\section{Additional files}

Additional file 1: The study protocol (2011-06) for the trial. (DOC 6959 kb)

Additional file 2: Appendices (inclusion and exclusion criteria of the trial; detailed methods for randomization; detailed methods for cell isolation, characterization and cell transplant preparation; and detailed methods for determining bone fill). (DOCX $2833 \mathrm{~kb}$ )

\section{Abbreviations \\ CAL: Clinical attachment level; GCP: Good Clinical Practice; GMP: Good Manufacturing Practice; GR: Gingival recession; GTR: Guided tissue regeneration; MSC: Mesenchymal stem/stromal cell; PD: Probing depth; PDL: Periodontal ligament; PDLSC: Periodontal ligament stem cell.}

\section{Competing interests}

The authors declare that they have no competing interests.

\section{Authors' contributions}

F-MC contributed to the study conception and design, data acquisition, analysis, and interpretation, and drafted and critically revised the manuscript. L-NG and B-MT contributed to the study design, data acquisition, analysis, and interpretation, and drafted and critically revised the manuscript. Y-JZ contributed to the study conception and design, 
data acquisition, analysis, and interpretation, and drafted the manuscript $X-Y Z, G-Y D, H L, Q C$, and JX contributed to the study conception, data acquisition and interpretation, and critically revised the manuscript. YYu, $\mathrm{R}-\mathrm{XW}$, and YYi contributed to data acquisition, analysis, and interpretation, and drafted the manuscript. SS and YJ contributed to the study conception and design, data acquisition, analysis, and interpretation, and critically revised the manuscript. All of the authors read and approved the manuscript.

\section{Authors' information}

F-MC, L-NG, and B-MT are co-first authors. F-MC, SS, and YJ are cocorresponding authors.

\section{Acknowledgements}

This project was supported by a translational research grant from the Fourth Military Medical University School of Stomatology. The funding bodies played no role in the study design or the decision to submit the manuscript for publication. The authors also acknowledge our previous basic and animal research in this field that was supported by grants from the National Natural Science Foundation of China (81471791, 81500853 and 81530050).

\section{Received: 25 September 2015 Revised: 14 December 2015}

\section{Accepted: 27 January 2016 Published online: 19 February 2016}

\section{References}

1. Burt B. Position paper: epidemiology of periodontal diseases. J Periodontol. 2005;76:1406-19.

2. Pihlstrom BL, Michalowicz BS, Johnson NW. Periodontal diseases. Lancet. 2005:366:1809-20.

3. Williams RC, Barnett AH, Claffey N, Davis M, Gadsby R, Kellett M, et al. The potential impact of periodontal disease on general health: a consensus view. Curr Med Res Opin. 2008:24:1635-43.

4. Chen FM, Zhang J, Zhang M, An Y, Chen F, Wu ZF. A review on endogenous regenerative technology in periodontal regenerative medicine. Biomaterials. 2010;31:7892-927.

5. Lu H, Xie C, Zhao YM, Chen FM. Translational research and therapeutic applications of stem cell transplantation in periodontal regenerative medicine. Cell Transplant. 2013;22:205-29.

6. Chen FM, Jin Y. Periodontal tissue engineering and regeneration: current approaches and expanding opportunities. Tissue Eng Part B Rev. 2010;16:219-55.

7. Reynolds MA, Aichelmann-Reidy ME, Branch-Mays GL. Regeneration of periodontal tissue: bone replacement grafts. Dent Clin North Am. 2010;54:55-71.

8. Villar CC, Cochran DL. Regeneration of periodontal tissues: guided tissue regeneration. Dent Clin North Am. 2010;54:73-92.

9. Cortellini P, Labriola A, Tonetti MS. Regenerative periodontal therapy in intrabony defects: state of the art. Minerva Stomatol. 2007;56:519-39.

10. Bosshardt DD, Sculean A. Does periodontal tissue regeneration really work? Periodontol 2000. 2009;51:208-19.

11. Trombelli L. Which reconstructive procedures are effective for treating the periodontal intraosseous defect? Periodontol 2000. 2005;37:88-105.

12. Chen FM, Sun HH, Lu H, Yu Q. Stem cell-delivery therapeutics for periodontal tissue regeneration. Biomaterials. 2012;33:6320-44.

13. Lin NH, Gronthos S, Mark BP. Stem cells and future periodontal regeneration. Periodontol 2000. 2009:51:239-51.

14. Bartold PM, McCulloch CA, Narayanan AS, Pitaru S. Tissue engineering: a new paradigm for periodontal regeneration based on molecular and cell biology. Periodontol 2000. 2000;24:253-69.

15. Catón J, Bostanci N, Remboutsika E, De Bari C, Mitsiadis TA. Future dentistry: cell therapy meets tooth and periodontal repair and regeneration. J Cell Mol Med. 2011;15:1054-65.

16. Seo BM, Miura M, Gronthos S, Bartold PM, Batouli S, Brahim J, et al. Investigation of multipotent postnatal stem cells from human periodontal ligament. Lancet. 2004;364:149-55.

17. Doğan A, Ozdemir A, Kubar A, Oygür T. Assessment of periodontal healing by seeding of fibroblast-like cells derived from regenerated periodontal ligament in artificial furcation defects in a dog: a pilot study. Tissue Eng. 2002;8:273-82

18. Doğan A, Ozdemir A, Kubar A, Oygür T. Healing of artificial fenestration defects by seeding of fibroblast-like cells derived from regenerated periodontal ligament in a dog: a preliminary study. Tissue Eng. 2003;9:1189-96.
19. Nakahara T, Nakamura T, Kobayashi E, Kuremoto K, Matsuno T, Tabata Y, et al. In situ tissue engineering of periodontal tissues by seeding with periodontal ligament-derived cells. Tissue Eng. 2004;10:537-44.

20. Bruckmann C, Walboomers XF, Matsuzaka K, Jansen JA. Periodontal ligament and gingival fibroblast adhesion to dentin-like textured surfaces. Biomaterials. 2005;26:339-46.

21. Yamada Y, Ueda M, Hibi H, Baba S. A novel approach to periodontal tissue regeneration with mesenchymal stem cells and platelet-rich plasma using tissue engineering technology: a clinical case report. Int J Periodontics Restorative Dent. 2006;26:363-9.

22. Yang Y, Rossi FM, Putnins EE. Periodontal regeneration using engineered bone marrow mesenchymal stromal cells. Biomaterials. 2010;31:8574-82.

23. Yang ZH, Zhang XJ, Dang NN, Ma ZF, Xu L, Wu JJ, et al. Apical tooth germ cell-conditioned medium enhances the differentiation of periodontal ligament stem cells into cementum/periodontal ligament-like tissues. J Periodontal Res. 2009;44:199-210.

24. Ding G, Liu Y, Wang W, Wei F, Liu D, Fan Z, et al. Allogeneic periodontal ligament stem cell therapy for periodontitis in swine. Stem Cells. 2010;28: 1829-38.

25. Park CH, Rios HF, Jin Q, Bland ME, Flanagan CL, Hollister SJ, et al. Biomimetic hybrid scaffolds for engineering human tooth-ligament interfaces. Biomaterials. 2010;31:5945-52.

26. Washio K, Iwata T, Mizutani M, Ando T, Yamato M, Okano T, et al. Assessment of cell sheets derived from human periodontal ligament cells: a pre-clinical study. Cell Tissue Res. 2010;341:397-404.

27. Tsumanuma Y, Iwata T, Washio K, Yoshida T, Yamada A, Takagi R, et al. Comparison of different tissue-derived stem cell sheets for periodontal regeneration in a canine 1-wall defect model. Biomaterials. 2011;32:5819-25.

28. Bright R, Hynes K, Gronthos S, Bartold PM. Periodontal ligament-derived cells for periodontal regeneration in animal models: a systematic review. J Periodontal Res. 2015;50:160-72.

29. d'Aquino R, De Rosa A, Lanza V, Tirino V, Laino L, Graziano A, et al. Human mandible bone defect repair by the grafting of dental pulp stem/progenitor cells and collagen sponge biocomplexes. Eur Cell Mater. 2009;18:75-83.

30. Feng F, Akiyama K, Liu Y, Yamaza T, Wang TM, Chen JH, et al. Utility of PDL progenitors for in vivo tissue regeneration: a report of 3 cases. Oral Dis. 2010;16:20-8.

31. McAllister BS. Stem cell-containing allograft matrix enhances periodontal regeneration: case presentations. Int J Periodontics Restorative Dent. 2011; 31:149-55.

32. Kitamura M, Nakashima K, Kowashi Y, Fujii T, Shimauchi H, Sasano T, et al. Periodontal tissue regeneration using fibroblast growth factor-2: randomized controlled phase II clinical trial. PLoS One. 2008;3:e2611.

33. Kitamura M, Akamatsu M, Machigashira M, Hara Y, Sakagami R, Hirofuji T, et al. FGF-2 stimulates periodontal regeneration: results of a multi-center randomized clinical trial. J Dent Res. 2011;90:35-40.

34. de Molon RS, Morais-Camillo JA, Sakakura CE, Ferreira MG, Loffredo LC, Scaf G. Measurements of simulated periodontal bone defects in inverted digital image and film-based radiograph: an in vitro study. Imaging Sci Dent. 2012:42:243-7.

35. Hynes K, Menicanin D, Gronthos S, Bartold PM. Clinical utility of stem cells for periodontal regeneration. Periodontol 2000. 2012;59:203-27.

36. Yoshida T, Washio K, Iwata T, Okano T, Ishikawa I. Current status and future development of cell transplantation therapy for periodontal tissue regeneration. Int J Dent. 2012;2012:307024.

37. Daley GQ, Scadden DT. Prospects for stem cell-based therapy. Cell. 2008; 132:544-8.

38. Chen FM, Zhao YM, Jin Y, Shi S. Prospects for translational regenerative medicine. Biotechnol Adv. 2012;30:658-72.

39. Lalu MM, McIntyre L, Pugliese C, Fergusson D, Winston BW, Marshall JC, et al. Safety of Cell Therapy with Mesenchymal Stromal Cells (SafeCell): a systematic review and meta-analysis of clinical trials. PLoS One. 2012;7: e47559.

40. Volponi AA, Pang Y, Sharpe PT. Stem cell-based biological tooth repair and regeneration. Trends Cell Biol. 2010;20:715-22.

41. Traini T, Valentini P, lezzi G, Piattelli A. A histologic and histomorphometric evaluation of anorganic bovine bone retrieved 9 years after a sinus augmentation procedure. J Periodontol. 2007;78:955-61.

42. Gantes B, Martin M, Garrett S, Egelberg J. Treatment of periodontal furcation defects. (II). Bone regeneration in mandibular class II defects. J Clin Periodontol. 1988;15:232-9. 
43. Mellonig JT. Histologic and clinical evaluation of an allogeneic bone matrix for the treatment of periodontal osseous defects. Int J Periodontics Restorative Dent. 2006;26:561-9.

44. Chen FM, Liu X. Advancing biomaterials of human origin for tissue engineering. Prog Polym Sci. 2016;53:86-168.

45. Kaigler D, Pagni G, Park CH, Braun TM, Holman LA, Yi E, et al. Stem cell therapy for craniofacial bone regeneration: a randomized, controlled feasibility trial. Cell Transplant. 2013;22:767-77.

46. Padial-Molina M, O'Valle F, Lanis A, Mesa F, Dohan Ehrenfest DM, Wang HL, et al. Clinical application of mesenchymal stem cells and novel supportive therapies for oral bone regeneration. Biomed Res Int. 2015;2015:341327.

Submit your next manuscript to BioMed Central and we will help you at every step:

- We accept pre-submission inquiries

- Our selector tool helps you to find the most relevant journal

- We provide round the clock customer support

- Convenient online submission

- Thorough peer review

- Inclusion in PubMed and all major indexing services

- Maximum visibility for your research

Submit your manuscript at www.biomedcentral.com/submit
Biomed Central 